\title{
Anticipatory prescribing for end of life care: a survey of community nurses in England
}

Wilson E, Seymour J, Seale C (2016) Anticipatory prescribing for end of life care: a survey of community nurses in England. Primary Health Care. 26, 9, 22-27. Date of submission: 6 April 2016; date of acceptance: II May 2016. doi I0.7748/phc.2016.ell5I

\section{Eleanor Wilson}

Senior research fellow, University of Nottingham School of Medicine

\section{Jane Seymour}

Professor of palliative and end of life care, School of Nursing and Midwifery, University of Sheffield

\section{Clive Seale}

Formerly professor of sociology, Brunel University

\section{Correspondence \\ eleanor.wilson@nottingham. ac.uk}

\section{Peer review}

This article has been subject to external double-blind peer review and checked for plagiarism using automated software

\section{Conflict of interest} None

\begin{abstract}
Anticipatory prescribing is increasingly common in the UK, yet little is known about nurses' roles in the process. As part of a wider study, a postal survey of 575 community or district, nursing home and palliative care nurses was undertaken in two regions. Responses appeared to show anticipatory prescribing as a common practice and, once in place, prescriptions were often used. Nurses reported taking prominent roles throughout the anticipatory prescribing process and principal responsibility for administering and monitoring medications. Many respondents recounted good working relationships with GPs. However, some reported issues including being challenged by GPs who were reluctant to prescribe medication, being given incorrect prescriptions and having difficulty in gaining access to the necessary medications. Qualitative comments indicated that being a nurse prescriber could enhance confidence to use anticipatory prescriptions. Importantly, respondents perceived that anticipatory prescriptions facilitated provision of good end of life care.
\end{abstract}

\section{Keywords}

anticipatory medication, anticipatory prescribing, community nursing, end of life care, nurses' perspectives, palliative care, survey, symptom management

\section{Introduction}

Medications prescribed, dispensed and held in a patient's home in anticipation of need are becoming increasingly used in the UK (Wilcock 2011). These 'just in case' or 'anticipatory' medications can be prescribed in advance to manage common symptoms at the end of life, including pain, nausea and vomiting, anxiety and excessive secretions. They are usually prescribed by a GP and dispensed and then held in the patient's home or nursing home for use when needed. Having immediate access to necessary medications in the home is regarded as a key means of improving symptom management and enabling better end of life care in the community (Carney 2011, Lawton et al 2012, Faull et al 2013, National Institute for Health and Care Excellence (NICE) 2015).

Anticipatory prescribing is seen as enabling professional staff, such as community and district nurses, to respond immediately to patients' needs (Carney 2011). Yet little is known about nurses' roles in and experiences of anticipatory prescribing (Eisenhauer et al 2007, Wilson and Seymour 2013), and this is cited as a recommended area of research in the recent NICE guideline on caring for the dying (2015). A synthesis of existing related research suggests that nurses may be challenged by a lack of resources, limited knowledge and experience with the relevant medications, and power differentials between themselves and medical practitioners (Wilson and Seymour 2013). Although local audits have been carried out to assess the implementation of anticipatory prescribing (Amass and Allen 2005, Carney and MacRobbie 2008, ScottAiton 2009, Carney 2011, Lawton et al 2012), there are no larger scale studies in existence.

Insights from a qualitative study by Faull et al (2013) suggest anticipatory prescribing is complex, and that healthcare staff have a range of interpretations of what is meant by prescribing in advance. Faull et al (2013) conclude that anticipatory prescribing is a process rather than a oneoff event, and highlight the need to build relationships between professionals to ensure good communication in teams and across organisational boundaries, including out-ofhours care.

This article reports the findings of a survey that elicited the views of 575 community or district, nursing home and palliative care nurses from two UK regions: the East Midlands and Lancaster/South Cumbria. The aim of the survey was to gain insight into the roles and experiences of a wide range of community nurses in end of life medication 
decisions. Quantitative and qualitative aspects of the survey are drawn on to give an overview of the key findings.

\section{Methods}

The survey was part of a three-phase, mixedmethods study to examine community nurses' experiences of working with anticipatory prescriptions. Phase one was a literature review of 26 studies from ten countries (Wilson and Seymour 2013). Phase two was a qualitative ethnographic study using interviews and observations (Wilson et al 2014, Wilson et al 2015). The survey was the third phase of the study, informed by the data gathered in the literature review and ethnography. The survey questionnaire was developed by the research team and supported by an advisory panel with expertise in community and end of life care nursing. A face validity exercise was undertaken with a group of district nurses from the East Midlands. The nurses completed the survey and gave feedback in a focus group on the wording, style, order and appropriateness of the questions. In light of their comments, adjustments were made to the questionnaire.

\section{Survey sample}

The survey was conducted in the East Midlands and Lancaster/South Cumbria. The Binley's database of NHS staff was used to select 500 district and community nurses at random in each area. In addition, all specialist palliative care nurses, Macmillan and Marie Curie nurses (grouped and referred to as 'palliative care nurses'), and managers and matrons of nursing homes were also selected in the two areas. It was recognised that the database would not be completely up-to-date and search terms might identify some nurses who were not working in the fields required. To minimise the number of questionnaires sent to inappropriate people, the lists were examined by hand by the study team. During this process, job and institutional titles were inspected to exclude individuals who were not likely to be involved in caring for adults who had died at home or in a nursing home. For example, we excluded all those located in hospitals or with job titles that would not have a primary focus on palliative care for adults.

\section{Data collection and management}

From February to May 2013 a postal questionnaire and two reminders were sent to 1,739 individuals; 181 mailings were returned as the individual no longer worked at the institution concerned, leaving a total valid sample of 1,558. Questionnaires were returned and the data was logged in a database, managed through the programme Statistical Package for the Social Sciences (SPSS).

\section{Data analysis}

Survey data was analysed to produce a series of descriptive statistics supported by the SPSS software. Responses were compared across the three categories of nurses (nursing home, community/district and palliative care).

The free text sections were analysed using a thematic analysis (Braun and Clarke 2006) to allocate codes to the comments and identify patterns. These codes were then grouped and distilled to generate themes. Themes were compared with the quantitative findings and are used in this article to add depth to those findings. As not all respondents answered all questions, total response rates for individual questions are reported and vary throughout the article.

\section{Ethical approval}

We obtained ethical approval from the National Research Ethics Service and governance approval from the NHS trusts at each research site.

\section{Findings}

The overall response rate was $37 \%$ $(n=575 / 1,558)$. While nursing home nurses $(49.6 \% ; n=231 / 466)$ and palliative care nurses (47.4\%; $n=151 / 318)$ responded well, a lower response from community/district nurses $(24.9 \% ; n=193 / 774)$ reduced the overall response rate.

Comparing the two regions, the East Midlands had a slightly better response rate (39.7\%; $n=380 / 957$ ) compared to Lancaster/ South Cumbria (32.4\%; $n=195 / 601)$. Respondents were predominantly female $(95 \% ; n=540 / 569)$, had worked as a nurse for 20 years or more $(64 \% ; n=366 / 572)$ and were likely to have some kind of additional training in palliative $(70 \% ; n=404 / 575)$ and/or end of life care $(78 \% ; n=440 / 575)$. Respondents reported attending a range of courses and training so it was not possible to compare the level or quality of the training. From the written comments, those with additional qualifications appeared to value this training with regard to the anticipatory prescribing process. Some of those with a prescribing qualification acknowledged that they could take on the prescription-writing role in the process:

'Community/district nurses know more about end of life drugs and care than GPs. Consequently the decision to prescribe

\section{Online archive}

For related information, visit our online archive and search using the keywords

This work was supported by the Marie Curie Research Grants Scheme, grant [C30627/Al2557] 
generally is dictated by the nurse and the GP will take their advice. Hopefully, all nurses will hold NISP (V300) [nurse independent and supplementary prescribers] qualification soon and prescribe for patients.' (District nurse team leader.)

'I feel that as an NISP I am extremely confident in prescribing for palliative care patients, specifically in end of life care.' (Palliative care nurse.)

Figure I. Prescription writing (539 responses)

'Doctors with whom I work are reluctant to provide anticpatory prescritions for drugs used in end of life care'

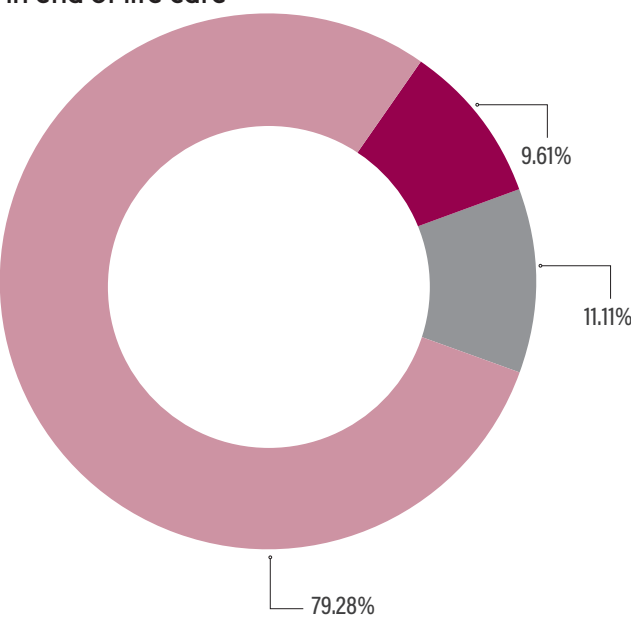

- Always or frequently

- Neither frequent nor infrequent

- Infrequently or never

TABLE I. Characteristics of patient cases reported by nurses to have received anticipatory prescription medications

\begin{tabular}{l|l|l|}
\hline Characteristic & Male & $\begin{array}{l}\text { Number of patient cases as reported by nurse } \\
\text { respondents (\%) }\end{array}$ \\
\hline Gender & Female & $194 / 442(43.9 \%)$ \\
\hline Age & Under 50 & $248 / 442(56.1 \%)$ \\
\hline Place of death & $50-69$ & $32 / 412(7.7 \%)$ \\
\hline & Ho-89 & $117 / 412(28.4 \%)$ \\
\hline Cause of death & Hospice & $263 / 412(63.9 \%)$ \\
\hline & Nursing/care home & $4 / 448(0.9 \%)$ \\
\hline & Cwn home & $12 / 448(2.7 \%)$ \\
\hline Cancer & $198 / 448(44.2 \%)$ \\
\hline & Heart disease & $234 / 448(52.2 \%)$ \\
\hline Stroke & $344 / 434(79.3 \%)$ \\
\hline & Other & $27 / 434(6.2 \%)$ \\
\hline & $12 / 434(2.8 \%)$ \\
\hline
\end{tabular}

Caring for patients at the end of life Respondents were asked how many patients they had cared for at the end of life in the past year. Overall, $83.7 \%(n=481 / 575)$ reported providing care to at least one person who had died in the past year. Of these 481 nurses, 471 (98.1\%) reported that 'an anticipatory prescription for drugs aimed at relieving symptoms and distress' was in place for at least one patient; and of those reporting this, $98.3 \%(n=463 / 471)$ said that in at least one case these prescriptions were used. Respondents reporting that in no cases were prescriptions used (just eight respondents), worked in care homes or in 'other' roles.

\section{Characteristics of patients}

Having answered some questions about anticipatory prescriptions, respondents were then asked to recall details of one specific patient for whom an anticipatory prescription had been used and to answer a series of further questions about that particular case. Table 1 gives a breakdown of the characteristics of these cases. Where patients' age categories were reported $(n=412), 63.8 \% \quad(n=263)$ were said to be aged 70 or over. The majority of the patients for whom the use of an anticipatory prescription was recalled were reported by nurses as dying in their own home or in a care home $(96.4 \% ; n=432 / 448)$. A primary cause of death was provided for 434 patient cases and in $79.3 \%(n=344 / 434)$ of these, cancer was reported by nurses as the registered cause of death. Fewer respondents from nursing homes reported that cancer was a cause of death among the patients whom they recalled as receiving anticipatory medications.

\section{Prescribing and dispensing}

Nurses reported working well with GPs and perceived that they had good access to the medications needed. Figure 1 shows that $79.2 \%$ $(n=427 / 539)$ of nurses reported that they 'infrequently or never' found doctors reluctant to prescribe anticipatory medication, and $11.1 \%(n=60 / 539)$ reported this as 'neither frequent nor infrequent'. But it was evident there was some variation. A small proportion, $9.6 \%(n=52 / 539)$, agreed that some doctors were reluctant to provide anticipatory prescriptions (Figure 1) and this point was reflected in the qualitative comments:

'I work with different GP practices. All very individual, some more knowledgeable than others. Some happy to listen to nursing home staff and families and take our opinions on board. I have to get assistance from a palliative care nurse at times to help me get a GP to prescribe.' (Matron in a nursing home.) 
'Not all practices in my location are happy to prescribe anticipatory medications, as they feel this is a waste of their budget and that out-of-hours doctors are there to be called upon for the prescribing of anticipatory drugs. In the main, the reluctance of GPs in prescribing is improving.' (Manager in a nursing home.)

Similarly, a few nurses $(8.6 \% ; n=45 / 525)$ said they 'always or frequently' experienced significant difficulties in obtaining the drugs specified in anticipatory prescriptions used in end-of-life care. For a further $11.2 \%(n=59 / 525)$ this was reported as 'neither frequent nor infrequent'. However, the majority of nurses $(80.2 \% ; n=421 / 525)$ said they encountered these difficulties 'infrequently or never' (Figure 2).

Across the three categories of nurses, slightly less than one fifth $(18.4 \% ; n=97 / 527)$ agreed with the statement 'Anticipatory prescriptions are incorrectly written up by doctors'.

However, when looking at the categories of respondents this was reported more frequently by palliative care nurses $(24.3 \% ; n=28 / 115)$ and community/district nurses $(25.7 \%$; $n=45 / 175)$ than by nursing home nurses $(9.7 \%$ $n=19 / 195$ ). Comments about these issues were also added in the written section of the survey:

'I have real problems with GPs prescribing [end of life] drugs, they are usually unable to estimate doses correctly, have no idea how to prescribe when a patient has a fentanyl patch, or is a slow release morphine. This weekend, in 1 day, two prescriptions were written incorrectly.' (Palliative care nurse.)

Figure 2. Dispensing (525 responses)

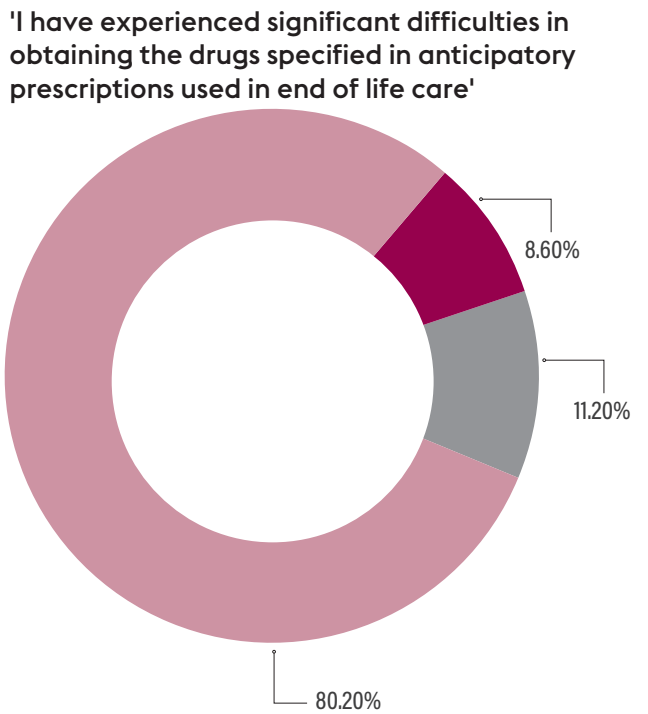

- Always or frequently

- Neither frequent nor infrequent

- Infrequently or never

\section{Anticipatory medications used}

Where respondents reported on the care of one specific patient who had been in receipt of anticipatory medications, midazolam was the drug most commonly reported to have been used in the last month of the patient's life. This applied to $81.9 \%(n=367 / 448)$ of the patients where use of an anticipatory prescription was recalled by nurses. This was followed by levomepromazine $(32.5 \% ; n=143 / 440)$ and then haloperidol $(23.9 \% ; n=104 / 435)$. Figure 3 shows the percentage of drugs used by category of respondent; nurses in all three categories reported similar patterns of usage.

\section{Managing the use of anticipatory} medications

Having the confidence to make decisions relating to the use of drugs specified in anticipatory prescriptions was considered to be important by $92 \%(n=483 / 525)$ of nurses responding to the survey. For example, when reporting the use of midazolam in specific patient cases, $82.2 \%(n=287 / 349)$ of the responding nurses felt they had responsibility for assessing that patient's response to the drug; $34.1 \%(n=119 / 349)$ felt this responsibility lay with the GP; and 3.2\% $(n=11 / 349)$ with the specialist palliative care doctor (for this question, respondents had the opportunity to select more than one option).

Table 2 shows that for these patient cases medications were either 'not increased' or just

Figure 3. Drugs used by respondent category

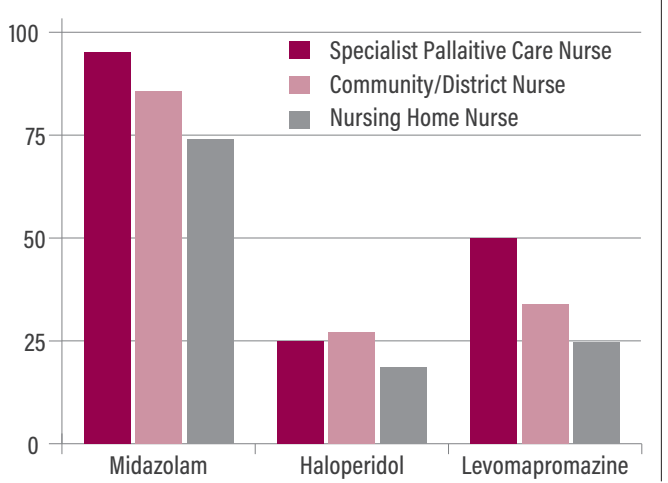

TABLE 2. Reported changes in dosages for patient cases in the last three days of life

\begin{tabular}{|l|l|l|l|}
\hline & Midazolam & Haloperidol & Levomepromazine \\
\hline No increase & $51.1 \%(n=165)$ & $54.0 \%(n=47)$ & $55.2 \%(n=69)$ \\
\hline Gradual increase & $42.1 \%(n=136)$ & $41.4 \%(n=36)$ & $40.0 \%(n=50)$ \\
\hline Strong increase last day & $6.8 \%(n=22)$ & $4.6 \%(n=4)$ & $4.8 \%(n=6)$ \\
\hline TOTAL (=100\%)* & 323 & 87 & 125 \\
\hline
\end{tabular}

*Patients may have received more than one of these drugs

\section{Write for us}

journals.rcni.com/r/ phc-author-guidelines 


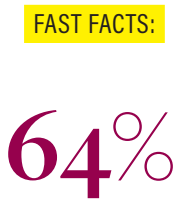

of respondents had been a nurse for 20 years or more

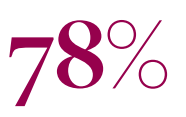

had training in end of life care

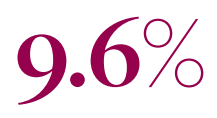

said doctors were reluctant to prescribe anticipatory drugs

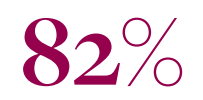

of nurses had medicine monitoring responsibility 'increased gradually' over the last three days of life, with a 'strong increase' on the last day being rare.

Nurses reported that the anticipatory medications successfully controlled those symptoms they were intended to relieve in $89.6 \%$ of the patient cases they recalled. In a more general sense, most respondents $(96.0 \%$; $n=504 / 525$ ) agreed that when anticipatory prescriptions were in place they 'always or frequently' enabled respondents to improve the quality of end of life care they provided:

'It helps to provide better quality of care for the patients, especially out of hours.' (District nurse.)

'Anticipatory prescribing is essential while medications are not always needed it gives peace of mind to the resident, relatives and staff that, should symptoms develop, medication is at hand to deal with them. Proactive decision making is essential in this area.' (Matron in a nursing home.)

\section{Discussion}

This article has reported on one aspect of a wider study to explore nurses' roles in the use of anticipatory prescriptions. A survey was used to elicit views from 575 community/ district, nursing home and palliative care nurses across two regions in England. Initially nurses were asked to report their views about anticipatory prescribing in general; they were then asked to refer to a specific patient whom they recalled had received anticipatory medications and to answer a series of further questions about that patient. The survey shows that the majority of nurses were able to report on at least one patient who had had an anticipatory prescription in place. Moreover, when in place these prescriptions were used. In line with Carney's (2011) audit of the use of 'just in case' boxes in Scotland, the majority of nurses felt that having anticipatory medications in place enabled them to provide better care and could improve the quality of death.

Our findings indicate that nurses have a number of pivotal and complex roles throughout the 'process' of anticipatory prescribing (Faull et al 2013). The survey findings support those of the qualitative work undertaken in the earlier part of the study and reported elsewhere (Wilson et al 2015).

Our findings give some insight into the types of medication used for symptom management and the pattern of their use. Of the 441 specific cases where nurses recalled that a patient had received anticipatory medications, midazolam was reported as the most commonly-used drug, with levomepromazine and then haloperidol used less often. These are often considered to be three of the 'core' drugs used in anticipatory prescriptions (Wilcock 2011). In the cases reported, it was rare for nurses to recall any sudden increase in dosage of the medications used in the last 3 days of life; any increases that were recalled were considered by respondents as 'gradual'.

Nurses responding to the survey reported a particular responsibility for monitoring the results of giving the medications, with GPs and other doctors involved less often. Narratives from nurses in the ethnographic element in phase two of the study, recounted that decisions about the use of anticipatory medications were primarily regarded as the responsibility of the healthcare team and often of the nurse (Wilson et al 2015).

Although for the most part nurses reported that relationships with doctors were working well, a small proportion of respondents reported difficulties, again echoing findings from the ethnography in phase two of the study (Wilson et al 2015). Some encountered reluctance in some doctors to provide anticipatory prescriptions. A small number of respondents stated the effectiveness of the process could be challenged by prescriptions that were incorrectly written by doctors or by difficulties in obtaining the drugs. These findings resonate with observations by Faull et al (2013) that there is potential for the process to stall at any stage. Guidance from NICE (2015) suggests that when considering the need for anticipatory medications clinicians should not only weigh up benefits and harms of prescribing in advance but should also recognise the possibility of sudden deterioration and the time it might take to obtain medications. Hence, the recommendation is that prescriptions are put in place as early as possible but remain under review (NICE 2015).

A small number of qualitative comments by responding nurses indicated that they valued being a nurse prescriber and that this had enhanced their confidence and capacity to provide good care. Webb and Gibson's (2011) audit of nurse independent prescribers supports this, concluding that prescribing by nurses is effective, timely and appropriate, especially during out-of-hours periods.

\section{Limitations}

Of the 1,739 (10.4\%) questionnaires sent to people listed by Binley's, 181 were returned as the person had moved on since the list was compiled. It seems likely that the response was somewhat skewed towards nurses with more experience, as this statistic suggests a time lag before Binley's picks up a name and adds it 
to their database. If true, there would tend to be an under-representation of people recently starting in posts, and this group may be more likely to contain nurses who had not worked in the role before. A high proportion of respondents with 20 years' or more experience may also indicate that the questionnaire was passed on to nurses with more experience to complete and/or that more experienced nurses were more likely to deal with patients with anticipatory prescriptions in place.

The survey was sent to nurses at a time when end of life care was under a great deal of scrutiny due to a media furore and the subsequent repeal of the Liverpool Care Pathway. This may have led to some nurses being reluctant to share their experiences of using anticipatory medications at the end of life, resulting in a lower response rate, particularly from community/district nurses.

It is important to note that we did not have direct access to patient data. Nurses were asked to report on one specific case in which they had been involved. We have no knowledge of how representative this case may have been of that respondent's caseload or how accurate was their reporting of medication use.

\section{Conclusion}

The survey offered insight into the roles and experiences of a substantial number and range of nurses working with end of life medications in the community. Survey responses appear to show anticipatory prescribing is common and once in place prescriptions are often used. The comments in the survey show that nurses value the provision of anticipatory prescriptions and in general believe them to improve the care they provide for their patients. Although for the most part relationships with doctors were reported by the nurse respondents as working well, a small proportion recorded difficulties when some doctors were reluctant to provide prescriptions, when prescriptions were incorrectly written up by doctors, or in obtaining drugs.

This suggests pharmacists, nurses and GPs need to work together to establish the most appropriate ways of facilitating this process. In qualitative comments several nurses indicated that they valued being a nurse prescriber and that this enhanced their confidence and capacity to provide good care. Increasing the number of nurse independent and supplementary prescribers may help to support the process of anticipatory prescribing.

\section{Implications for practice}

"Nurses take on a number of pivotal and complex roles in the process that need to be supported by the wider clinical team.

" Multidisciplinary working is essential to establish the most appropriate ways to facilitate the anticipatory prescribing process.

"Increasing the number of nurse independent and supplementary prescribers may help support the process of anticipatory prescribing.

"Work to roll out training and resources to support anticipatory prescribing in the community should continue while being mindful of the time constraints faced by community health professionals.
FURTHER RESOURCES

The Liverpool Care Pathway can be accessed at gov.uk/government/ publications/review-ofliverpool-care-pathwayfor-dying-patients

\section{References}

Amass C, Allen M (2005) How a 'just in case' approach can improve out-of-hours palliative care. Pharmaceutical Journal. 275, 22-23.

Braun V, Clarke V (2006) Using thematic analysis in psychology. Qualitative Research in Psychology. 3, 2,77-101.

Carney J (2011) Analysis of 'Just in Case' Anticipatory Audit Forms 1st December 2010-28th February 2011. Palliative Care Network and NHS Highland, Inverness.

Carney J, MacRobbie A (2008) Analysis of 'Just in Case' Anticipatory Prescribing Pilot. Final Report. Palliative Care Network and NHS Highland, Inverness.
Eisenhauer L, Hurley A, Dolan N (2007) Nurses' reported thinking during medication administration. Journal of Nursing Scholarship. $39,1,82-87$

Faull C, Windridge K, Ockleford E et al (2013) Anticipatory prescribing in terminal care at home: what challenges do community health professionals encounter? BMJ Supportive \& Palliative Care. 3, 91-97.

Lawton S, Denholm M, Macaulay L et al (2012) Timely symptom management at end of life using 'just in case' boxes. British Journal of Community Nursing. 17, 4, 182-183.
National Institute for Health and Care Excellence (2015) Caring for the Dying Adult: NICE Guideline. NICE, London

Scott-Aiton A (2009) Creating a tool to improve anticipatory prescribing in palliative care Pharmaceutical Journal. 283, 283-284.

Webb W, Gibson V (2011) Evaluating the impact of nurse independent prescribing in a weekend clinical nurse specialist service. International Journal of Palliative Nursing. 17, 11, 537-543.

Wilcock A (2011) Pre-emptive prescribing in the community. In Twycross R, Wilcock A (Eds) Palliative Care Formulary (PCF4). Fourth edition. Palliativedrugs.com, Nottingham.
Wilson E, Morbey H, Brown J et al (2015) Administering anticipatory medications in end-oflife care: a qualitative study of nursing practice in the community and in nursing homes. Palliative Medicine. 29, 1, 60-70.

Wilson E, Seymour J (2013) Understanding the role of nurses in the management of symptoms and distress in the last days of life. In Sterckx S, Raus $\mathrm{K}$, Mortier F (Eds) Continuous Sedation at the End of Life. Cambridge University Press, Cambridge.

Wilson E, Seymour J, Morbey H et al (2014) Anticipatory prescribing in end-of-life care. Nursing Times. 110, 44, 16-17. 